\title{
LE CONTROLE DE LA DATE DE LA REPRODUCTION CHEZ LA BREBIS ET LA VACHE
}

\author{
PAR \\ L. DAUZIER $\left({ }^{(}\right)$ \\ Station de Physiologie animale, C.N.R.Z., Jouy-en-Josas.
}

I es races ovines élevées dans notre pays présentent le plus souvent une période d'activité sexuelle s'étendant de la fin de l'été à l'hiver. En effet, si certaines races ont dans leur zone naturelle d'élevage une période de repos sexuel peu marquée (Préalpes-du-Sud, Bizet), on constate souvent chez les races où pour des raisons économiques on pratique la lutte de printemps, une stérilité relative, une certaine proportion des femelles ne devenant pas gestantes (Ile-de-France) à cette époque de l'année. I e contrôle de la date d'accouplement se présente donc sous deux aspects :

-- groupage du rut pendant la période d'activité sexuelle (fin de l'été-hiver) ;

- déclenchement des cycles sexuels pendant l'ancestrus (fin de 1'hiver-été).

Comme l'indiquent les statistiques des centres d'Insémination artificielle, seul le premier aspect est essentiel chez la Vache, car il n'existe pas d'anœstrus généralement marqué.

\section{I. - LE GROUPAGE DU RUT CHEZ LA BREBIS ET CHEZ LA VACHE}

Le groupage des ruts d'un troupeau pendant une courte période peut présenter un intérêt économique, scientifique ou simplement pratique.

- Brebis soumises à la traite, production d'agneaux à date fixe ;

- Vaches inséminées avec le même échantillon de semence : taureaux de testage, sperme d'importation;

- Production laitière ou croissance de jeunes que l'on veut étudier comparativement en les plaçant dans les mêmes conditions saisonnières.

Dutt et Casida (I948), O'Mary, Pope et Casida (I950) ont montré les premiers, que des injections quotidiennes de ro $\mathrm{mg}$ de progestérone

(1) Avec la collaboration technique de J. P. Vincenst et Eliane Callanouin. 
inhibent, chez la plupart des brebis traitées, le rut et l'ovulation qui réapparaissent 2 à 3 jours après la fin du traitement.

Nous avons confirmé ces résultats et montré que les réponses des Brebis n'étaient pas indifférentes à la dose selon leur race (DAUZIER, OrTavant, Thibault et Wintenberger, I953).

Deux points importants restaient donc à préciser : la généralisation aux différentes races, et la simplification de la méthode, condition essentielle de son application.

$I^{0}$ Nous avions eu l'occasion d'observer des réponses différentes selon la race, soit avec la progestérone, soit avec 1'hormone gonadotrope (Dauzier, Ortavant, Thibault et Wintenberger, I953).

Nous avons donc comparé les réactions de brebis Boukhara, Ile-deFrance, Lacaune et Texel aux injections de progestérone.

Il ressort du tableau I que (conformément aux résultats de Dur's' et CASIDA), la plupart des brebis qu'elles soient Boukhara, Lacaune on Texel,ont eu un rut décalé et groupé 2 à 4 jours après la dernière injection de ro mg. Par contre, 3 brebis lle-de-France seulement sur 6 traitées, ont eu un œstrus décalé avec les mêmes doses de progestérone.

TABLEAU I

\begin{tabular}{|c|c|c|c|c|c|}
\hline $\begin{array}{l}\text { Dose quotidienne } \\
\text { de progestérone } \\
\text { injectée en } \\
\text { solution huileuse } \\
\text { (it jours) }\end{array}$ & $\begin{array}{c}\text { Race des } \\
\text { brebis } \\
\text { traitées }\end{array}$ & $\begin{array}{c}\text { Nombre de } \\
\text { brebis } \\
\text { traiteses }\end{array}$ & $\begin{array}{c}\text { Nombre de } \\
\text { brebis } \\
\text { dont l'oes- } \\
\text { trus est } \\
\text { suspendu }\end{array}$ & $\begin{array}{c}\text { Nombre de } \\
\text { brebis à } \\
\text { rut décalé }\end{array}$ & $\begin{array}{l}\text { Intervalle fin } \\
\text { traitement - rut } \\
\text { wltérieur pour les } \\
\text { brebis qui ont } \\
\text { le rut décalé }\end{array}$ \\
\hline $10 \mathrm{mg}$ & Boukhara & $i$ & 7 & 7 & 3,2 jours \\
\hline $10 \mathrm{mg}$ & Lacaune & 8 & 8 & 7 & 3,2 jours \\
\hline I0 $\mathrm{mg}$ & Texel (1) & 42 & 38 & 34 & 3,5 jours \\
\hline $10 \mathrm{mg}$ & Ile-dle-France & 6 & 3 & 2 & 3 jours \\
\hline $15 \mathrm{mg}$ & Ile-de-France & 8 & $\ddot{8}$ & 8 & 3,9 jours \\
\hline
\end{tabular}

(1) Pourcentage de Brebis gestantes de 74 p. roo apres saillie au premier rut.

Comme nous 1'avions déjà constaté (I953) il faut atteindre $15 \mathrm{mg} / \mathrm{jour}$ avec les brebis Ile-de-France pour obtenir le blocage du cycle ostrien, alors qu'avec les autres races utilisées, Io mg suffisent.

Après l'arrêt des injections, l'œestrus reparait et le décalage de l'ostrus ainsi provoqué est maintenu: les brebis sont à nouveaul en rut I 6 à $I 8$ jours plus tard et les cycles successifs restent déphasés par rapport aux cycles initiaux.

Notons enfin qu'après ce traitement la fécondité n'est pas affectée, ce qui permet l'utilisation de cette technique à des fins expérimentales comme ce fut le cas pour les brebis de race Texel (tableau I).

$2^{\circ} \mathrm{L}$ a multiplicité des injections étant incompatible avec les exigences de l'élevage, il était nécessaire d'essayer de mettre au point une méthode exigeant un petit nombre d'opérations. 
Dans ce but nous avons utilisé des implants de progestérone déposés sous la peau de brebis traitées à différents moments du cycle œestrien et retirés $\mathrm{I} 4$ jours après leur dépôt. Aucun résultat positif n'a été obtenu avec des implants de $200 \mathrm{mg}$ (tableau II).

\section{TABLEAU II}

\begin{tabular}{|c|c|c|c|c|c|c|}
\hline \multirow{2}{*}{$\begin{array}{l}\text { Int. jour } \\
\text { de l'im- } \\
\text { plantation } \\
\text { rut anté- } \\
\text { rieur }\end{array}$} & \multirow{2}{*}{$\begin{array}{l}\text { Quantité de } \\
\text { progestérone } \\
\text { implantée et } \\
\text { (nbre implants.) }\end{array}$} & \multirow{2}{*}{$\begin{array}{l}\text { Nomb. } \\
\text { de } \\
\text { brebis } \\
\text { traitées }\end{array}$} & \multirow{2}{*}{$\begin{array}{l}\text { Nomb. } \\
\text { Brebis } \\
\text { à œes- } \\
\text { trus } \\
\text { décalé }\end{array}$} & \multirow{2}{*}{$\begin{array}{l}\text { Int. entre le } \\
\text { jour de l'en- } \\
\text { lèvement } \\
\text { de l'im- } \\
\text { plant et le } \\
\text { rut suivant, } \\
\text { pour brebis } \\
\text { à cestrus } \\
\text { décalé }\end{array}$} & \multicolumn{2}{|c|}{$\begin{array}{c}\text { Intervalle (en jours) entre les } 2 \text { ruts } \\
\text { successifs }\end{array}$} \\
\hline & & & & & $\begin{array}{l}\text { Brebis à rut } \\
\text { décalé }\end{array}$ & $\begin{array}{l}\text { Brebis à rut } \\
\text { non décalé }\end{array}$ \\
\hline Int. $\leqslant 7 \mathrm{j}$. & $200 \mathrm{mg}(2)$ & 4 & o & & & $17-53-17-19$ \\
\hline Int. $>7 \%$ & $200 \mathrm{mg}(2)$ & 4 & $\circ$ & & & $17-19-34-17$ \\
\hline Int. $\leqslant 7 j$. & $400 \mathrm{mg}(4 \& 6)$ & I4 & IO & $\mathbf{I}, 8$ jour & $\begin{array}{c}(20-22-22-22- \\
21-20-2122-22 \\
(\text { moy. : } 21,5)\end{array}$ & $16-17-17-17$ \\
\hline Int. $>7 \mathrm{j}$. & $400 \mathrm{mg}(4 \& 6)$ & $2 \mathrm{I}$ & 4 & 2 jours & $\begin{array}{c}(29-28-28-24 \\
(\text { mov : } 27)\end{array}$ & $\begin{array}{l}16-36-32-17- \\
19-17-17-19- \\
17-17-17-17- \\
17-17+2 \text { anost. }\end{array}$ \\
\hline Int. $\leqslant 7 \mathrm{j}$. & $600 \mathrm{mg}(6 \& 8)$ & 6 & 4 & $\mathrm{I}, 6$ jour & $20-22-23-2 \mathrm{Ij}$ & $17-\mathrm{x} 6$ \\
\hline Int. $>7 \mathrm{j}$. & $600 \mathrm{mg}(6 \& 8)$ & 6 & o & & & $\begin{array}{l}\text { I } 8-16-17-18-19 \\
I \text { anostrus }\end{array}$ \\
\hline
\end{tabular}

Une forte proportion de brebis traitées avec 400 et $600 \mathrm{mg} \mathrm{le} 7^{\mathrm{e}}$ jour du cycle sexuel ou avant, ont eu un rut groupé et décalé, les réponses étant d'ailleurs assez inconstantes, mais seulement 4 résultats positifs sur 6 ont été obtenus avec des implantations postérieures au $7^{\mathrm{e}}$ jour du cycle.

L'adjonction d'une injection de 20,40 ou $60 \mathrm{mg}$ de progestérone en solution huileuse par voie sous-cutanée, le jour de l'implantation, n'a pas amélioré les résultats précédents.

NELLOR et COLE (I955) ayant signalé la possibilité de grouper l'œstrus de plusieurs vaches en utilisant une seule injection sous-cutanée de progestérone en suspension aqueuse, nous avons eu recours à la même méthode chez des brebis en utilisant des doses variables de progestérone et pour une même dose, un volume variable de diluant pour obtenir une dispersion différente des micro-cristaux de progestérone. I1 est en effet infiniment plus facile de faire une injection que de déposer un implant difficile à enlever car il peut s'enkyster ou provoquer des suppurations.

$\mathrm{Si}$ avec $50 \mathrm{mg}$, les résultats sont négatifs, on constate qu'avec des doses de Ioo mg dans Io cc on obtient la suspension de l'œstrus chez tous les animaux : malheureusement l'œstrus n'est pas toujours décalé (4 fois sur 7), certaines brebis n'ayant pas de rut dans les 3 mois siuvant le traitement et entrant vraisemblablement en anœstrus. Avec cette méthode 
les résultats sont donc inconstants ; il ne semble pas qu'en augmentant 1a dose d'hormone injectée on puisse espérer une amélioration des résultats. En effet, Neilor et COLE (I955) ont obtent1 chez la Vache letrs meilleurs résultats avec $560 \mathrm{mg}$ de progestérone, 700 à I I $20 \mathrm{mg}$ donnant des résultats plus aléatoires.

\section{TABLEAU III}

\begin{tabular}{|c|c|c|c|c|c|}
\hline $\begin{array}{c}\text { Quantité } \\
\text { progestérone injectée } \\
\text { volume diluant }\end{array}$ & $\begin{array}{c}\text { Numéro } \\
\text { brebis }\end{array}$ & $\begin{array}{l}\text { Intervalle } \\
\text { rut antér. } \\
\text { - injection }\end{array}$ & $\begin{array}{l}\text { Intervalle } \\
\text { Injection } \\
\text { - Rut }\end{array}$ & $\begin{array}{l}\text { Intervalle } \\
\text { ruts } \\
\text { successifs }\end{array}$ & $\begin{array}{l}\text { Nombre } \\
\text { brebis à rut } \\
\text { décalé }\end{array}$ \\
\hline $50 \mathrm{mg} / \mathrm{Icc}$ & $\begin{array}{r}837 \\
888 \\
5657 \\
870 \\
8803 \\
4508 \\
877 \\
458 \\
814 \\
2183\end{array}$ & $\begin{array}{l}2 \text { jours } \\
3 \\
3 \\
4 \\
5 \\
9 \\
\text { I0 } \\
\text { 12 } \\
\text { 12 } \\
\text { I3 }\end{array}$ & $\begin{array}{l}\text { II jours } \\
\text { I4 } \\
\text { I4 } \\
\text { II } \\
\text { I3 } \\
25 \\
7 \\
25 \\
25 \\
21\end{array}$ & $\begin{array}{l}13 \text { jours } \\
17 \\
17 \\
15 \\
18 \\
34 \\
17 \\
35 \\
37 \\
34\end{array}$ & $0 / 10$ \\
\hline $100 \mathrm{mg} / \mathrm{ICc}$ & $\begin{array}{r}4499 \\
866 \\
863\end{array}$ & $\begin{array}{l}2 \\
3 \\
3\end{array}$ & $\begin{array}{ll} & \text { ano: } \\
\text { I4 } & \\
\text { I3 } & \end{array}$ & $\begin{array}{l}17 \\
16\end{array}$ & \multirow[b]{2}{*}{$3 / 9$} \\
\hline & $\begin{array}{l}\mathrm{r} 57 \\
928 \\
887 \\
844 \\
448 \\
677\end{array}$ & $\begin{array}{r}3 \\
8 \\
\text { I0 } \\
\text { I I } \\
\text { I } 2 \\
\text { I3 }\end{array}$ & $\begin{array}{ll} & \text { anos } \\
12 & \\
24 & \\
24 & \\
\text { IO } & \\
\text { I I } & \end{array}$ & $\begin{array}{l}20 \\
34 \\
35 \\
22 \\
24\end{array}$ & \\
\hline IOO mg/rocc & $\begin{array}{r}938 \\
960 \\
834 \\
7501 \\
170 \\
934 \\
986\end{array}$ & $\begin{array}{r}8 \\
10 \\
10 \\
12 \\
13 \\
14 \\
14\end{array}$ & $\begin{array}{l}\text { anœe } \\
\text { anœs }\end{array}$ & $\begin{array}{l}22 \\
35 \\
25 \\
20 \\
24\end{array}$ & $4 / 7$ \\
\hline
\end{tabular}

\section{TABLEAU IV}

\begin{tabular}{|c|c|c|c|c|c|}
\hline $\begin{array}{l}\text { Numéro } \\
\text { Jvache }\end{array}$ & $\begin{array}{l}\text { Quantité } \\
\text { progestérone } \\
\text { injectée et } \\
\text { volume }\end{array}$ & $\begin{array}{l}\text { Intervalle } \\
\text { rut antérieur } \\
\ldots \text { injection }\end{array}$ & $\begin{array}{c}\text { Intervalle } \\
\text { injection } \\
\text { rut posté- } \\
\text { rieur }\end{array}$ & $\begin{array}{l}\text { Intervalle } \\
\text { entre les } \\
2 \text { ruts }\end{array}$ & Résultat \\
\hline $7 \ldots$ & $350 \mathrm{mg}(7 \mathrm{cc})$ & 6 jours & I5 jours & 21 jours & $\begin{array}{l}\text { Accouplée et non ges- } \\
\text { tante }\end{array}$ \\
\hline $3 \ldots \ldots$ & $350 \mathrm{mg}(7 \mathrm{cc})$ & 7 jours & I9 jours & 26 jours & $\begin{array}{l}\text { Abattue } 4 \text { jours après } \\
\text { saillie. Follicule se lu- } \\
\text { téinisant, pas ovulé }\end{array}$ \\
\hline I $4 \ldots$ & $550 \mathrm{mg}$ (I I cC) & I3 jours & I9 jours & $3^{2}$ jours & $\begin{array}{l}\text { Abattue I jours après } \\
\text { saillie } \\
\text { (non gestante) }\end{array}$ \\
\hline $6 \ldots$ & $55^{\circ} \mathrm{mg}$ (I I cc) & I5 jours & I 4 jours & 29 jours & $\begin{array}{l}\text { Abattue } 4 \text { j. après coït. } \\
\text { Follicule se lutéinisant, } \\
\text { pas ovulé. }\end{array}$ \\
\hline
\end{tabular}


Si 1'ovulation consécutive au traitement se produit normalement chez la Brebis, la fertilité n'étant pas affectée, il ne semble pas en être toujours de même chez la Vache puisque deux animaux abattus 4 jours après la fin du rut avaient des follicules qui se lutéinisaient sans avoir éclaté, et que deux autres vaches sacrifiées II jours après la fin de l'œstrus n'étaient pas gestantes.

On peut donc dire que dans les conditions actuelles, seules des injections quotidiennes et répétées de progestérone permettent d'obtenir chez la Vache et la Brebis un groupage de rut satisfaisant et une fertilité compatible avec les exigences de l'élevage ou de l'expérimentation.

\section{II. - DÉCLENCHEMENT DES CYCLES SEXUELS CHEZ LA BREBIS PENDANT L'ANCESTRUS}

L'intérêt d'une telle méthode est évidente pour les éleveurs désireux d'avoir un agnelage groupé, leur assurant l'écoulement des agneaux aux cours les plus élevés, ou facilitant la vente du lait de toutes les brebis dès l'ouverture de la laiterie.

L'idée d'utiliser la progestérone, seule ou associée avecl'hormone gonadotrope pour déclencher 1'œstrus pendant la période de repos sexuel découle des observations d'EveretT (r948) chez la Rate, puis de l'école de Casida sur la Brebis, la Truie et la Vache. Partant de ces observations, Du'T'T (I952) et DAUZIER et WINTENBERGER (I952), puis ROBINSON (I954 et I956) et enfin GORDON (I958) montraient que la progestérone seule, ou avec l'hormone gonadotrope, permettait d'obtenir l'œstrus suivi de la fécondation chez plus de la moitié des brebis traitées. Analysant le nombre d'injections de progestérone, la dose d'hormone gonadotrope, les délais optima entre les injections donnant les meilleurs résultats, DauzIer, Or'Tavan'T, Thibault et WinTENBERGer (I954) adoptèrent la technique simple suivante : deux injections sous-cutanées de $40 \mathrm{mg}$ de progestérone en solution huileuse à 3 jours d'intervalle, et trois jours plus tard, une injection de I 200 u. i. d'hormone gonadotrope sérique $\left({ }^{1}\right)$. L'utilisation de cette méthode leur permit de déclencher le rut chez 54 des 60 brebis traitées, 37 d'entre elles mettant bas 44 agneaux.

A partir de ces résultats, nous avons procédé à différents essais sur un troupeau expérimental d'une part, sur des troupeaux appartenant à des éleveurs d'autre part.

\section{Résultats d'essais sur un troupeau expérimental.}

Ce troupeau est élevé dans des conditions aussi proches que possible de celles des élevages normaux, les animaux subissant toutefois

(1) Hormone Gonadotrope Roussel. Nous en profitons pour remercier les Lits Roussel de l'aide apportée pour la fourniture des hormones.

Annales de Zootechnie.-- $195^{8}$. 
un certain nombre de manipulations (détection des chaleurs, pesée d'agneaux).

TABIALAU V

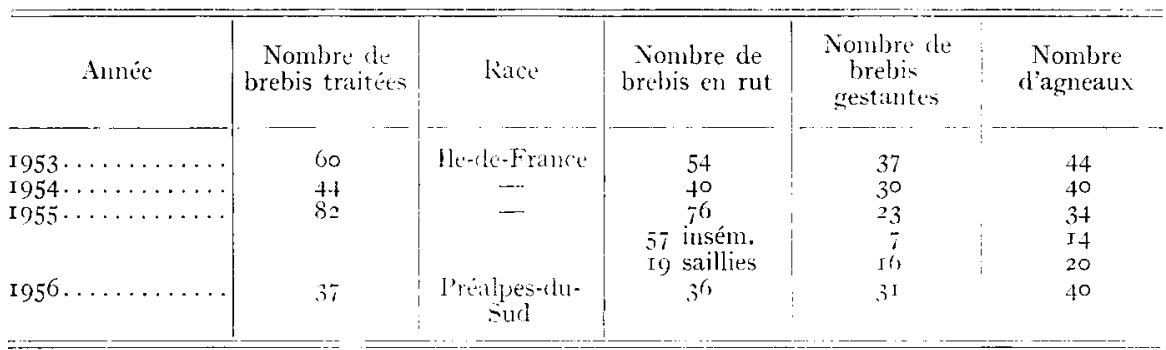

Un certain nombre de brebis en anostrus furent ainsi traitées de I953 à 1956 (tablean V). Ces résultats confirment pleinement ceux déjà publiés en 1954 .

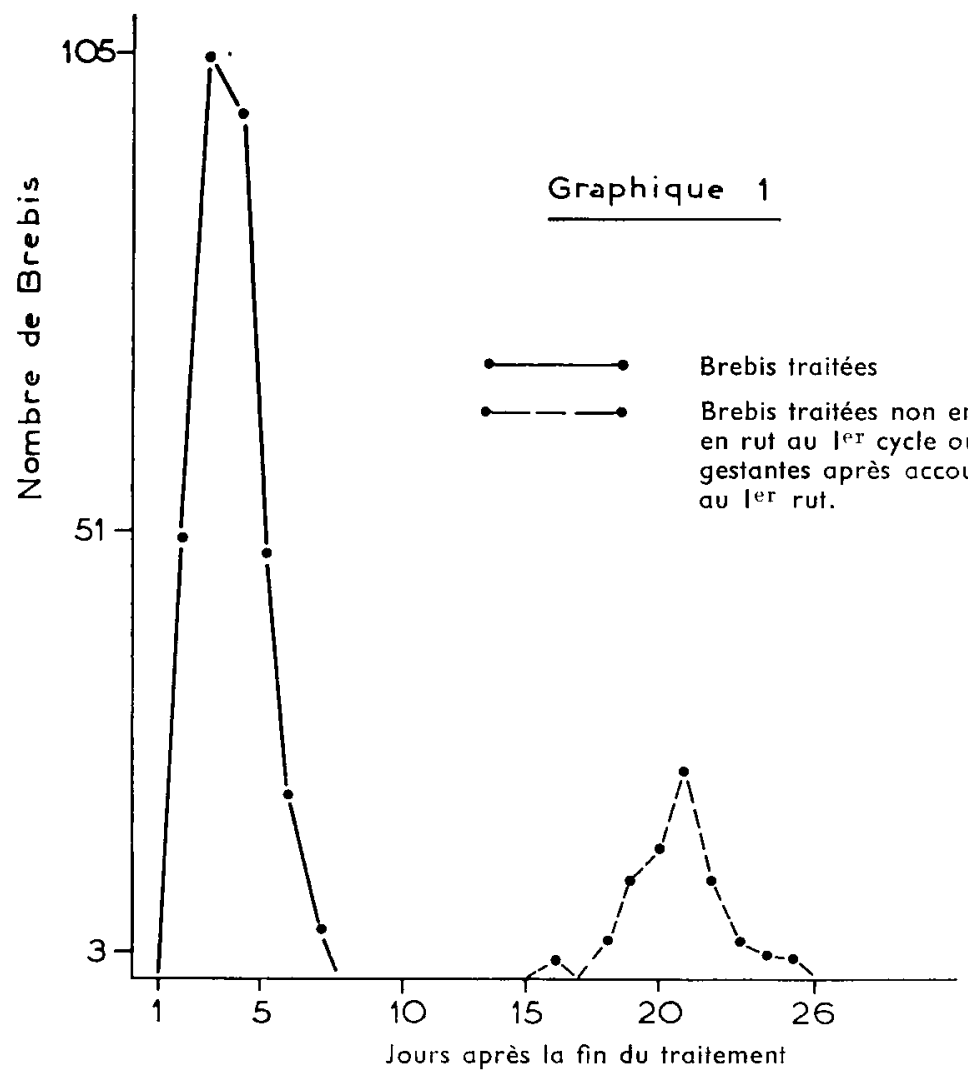

I,e graphique I montre nettement le groupage du rut consécutif anx injections de progestérone, le pluss grand nombre de brebis étant en rut 2 et 3 jours après la dernière intervention. Il apparait aussi que l'œestrus 
ainsi déclenché se reproduit $I^{6}$-I 7 jours plus tard si les brebis accouplées ne sont pas gestantes. Dans certains cas on constate même que des brebis qui n'entrent pas en rut, bien qu'ayant ovulé, après le traitement, présentent un oestrus au cycle suivant, c'est-à-dire de I9 à 2 I jours après la dernière injection.

On pouvait évidemment se demander si l'utilisation de substances hormonales ne risquait pas de créer ultérieurement des déséquilibres graves dans la reproduction des animaux traités. Les résultats obtenus en utilisant les mêmes brebis deux ou trois années consécutives sans permettre de répondre catégoriquement, ne montrent pas de variation dans l'efficacité du traitement (cité dans THIBACL'T et IACZIER, I956).

\section{TABLEAU VI}

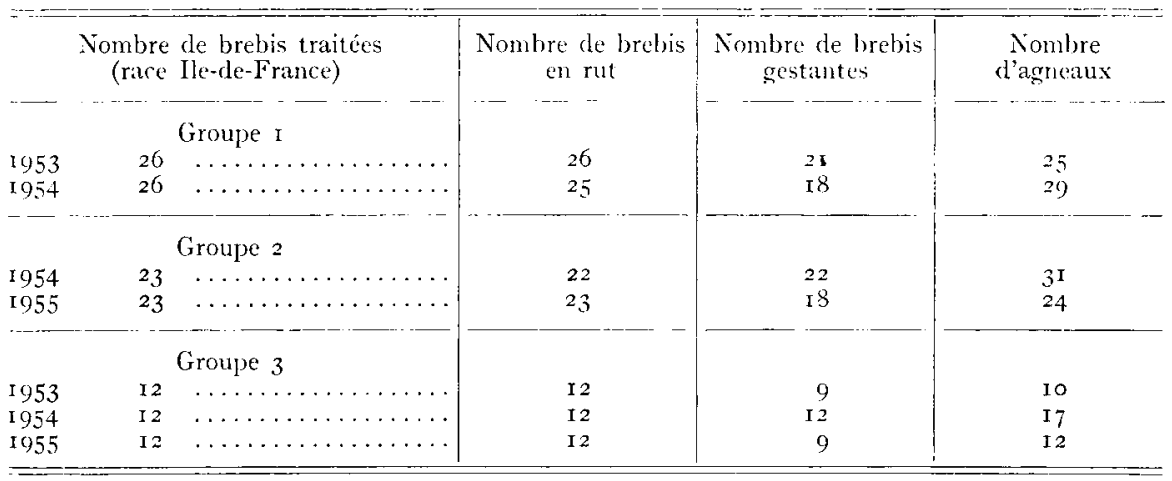

Notons enfin que le nombre moyen d'agneaux par mère est de I,3 quelques brebis ayant donné 3 et même 4 agneaux.

\section{go Résultats obtenus sur des troupeaux appartenant à des éleveurs.}

A la suite des essais très satisfaisants indiqués ci-dessus nous avons traité des brebis de différentes races, élevées dans des régions et des conditions souvent très différentes. Le tableau VII résume les résultats obtenus sur 642 brebis.

Il apparaît que certains résultats excellents en côtoient de très mauvais; sans pouvoir expliquer les causes de ces variations, on peut faire plusieurs constatations.

a) En jugeant la valeur du traitement uniquement sur l'apparition ou l'absence d'œstrus, on constate que dans les lots où le rut a été très soigneusement détecté sous contrôle d'un technicien qualifié, le nombre de brebis en cestrus à la suite des traitements est généralement satisfaisant (Lots B, D, E, I i), à l'exception des troupeaux A et G. Par contre, dans les troupeaux (non soulignés sur le tableau) où la surveillance de la détection des animaux en rut a été confiée aux bergers, la variabilité 
est beaucoup plus grande. Exemple, les troupeaux $I_{t}$ et $\mathrm{P}$ : respectivement 3 brebis en rut sur 25 et 20 traitées. On voit qu'une des premières conditions de succès de cette technique réside dans le soin avec lequel la recherche des brebis en cestrus est faite.

TABLEAT VII ( $\left.{ }^{1}\right)$

\begin{tabular}{|c|c|c|c|c|c|}
\hline$\ldots$ & $\begin{array}{l}\text { Date demiere } \\
\text { injection }\end{array}$ & $\begin{array}{c}\text { Race et type } \\
\text { d'élevare }\end{array}$ & $\begin{array}{c}\text { Xombre brebis } \\
\text { traités }\end{array}$ & $\begin{array}{c}\text { Nombre brebis } \\
\text { en rut } \\
\end{array}$ & $\begin{array}{l}\text { Nombre brebis } \\
\text { ayant mis-bas }\end{array}$ \\
\hline$A$ & Is avril 1955 & $\begin{array}{l}\text { lrompeau ambu- } \\
\text { lant, pas de race } \\
\text { diefinie. }\end{array}$ & ot brebis & $\begin{array}{l}\text { 56, dont } \\
\text { 30 sitillies }\end{array}$ & 7 \\
\hline$B$ & 20 avril s955 & $\begin{array}{l}\text { Lacaune } \\
\text { Procluction du lait. }\end{array}$ & 7. $\overline{\text { brebis }}$ & 13 & io \\
\hline$C$ & Io juin 1955 & $\begin{array}{l}\text { Me-de-France } \\
\text { Berigerie. }\end{array}$ & to brebis & 22 & 22 (30 agncitux) \\
\hline D) & 6 avril 10,56 & $\begin{array}{c}\text { Lacaune } \\
\text { (Productionde lait.) }\end{array}$ & s.t antenaises & $-\cdots$ & $30\left(4^{8}\right.$ agneaux) \\
\hline 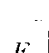 & $--\cdots$ & - - - & - & ${ }_{16} \cdot \quad--$ & - - \\
\hline $\begin{array}{l}E \\
--\end{array}$ & 6 avril $195^{6}$ & $-\quad--$ & ti brebis & $\begin{array}{l}4^{6} \\
-\end{array}$ & $39(53 \operatorname{agnc}(u x)$ \\
\hline$F$ & 28 févr. 1050 & $\cdots$ & 24 antenaises & 2,3 & + (4 aqneaux) \\
\hline$G$ & 16, jan1s. $145^{6}$ & Bizet & $\begin{array}{l}10.5 \text { dont } \\
15 \text { antenaise's }\end{array}$ & $\begin{array}{c}5^{8} \text { dont } \\
37 \text { sallies } \\
\text { sûres } \\
\text { I5 suillics } \\
\text { probables }\end{array}$ & I I \\
\hline II & 18 avril $105^{\circ}$ & Lillaume et races & 20 & 20 & 8 \\
\hline I & 18 avril I956 & locales & .30 & 29 & 25 \\
\hline$J$ & 18 avril 1956 & & .30 & 20 & + \\
\hline $\mathrm{K}$ & 17 avril 1050 & & 26 & I 8 & 1 \\
\hline I. & 17 avril 1050 & & 25 & 3 & 3 \\
\hline II & I7 avril 1050 & & 28 & 23 & I 3 \\
\hline$x$ & Is avril 1056 & & (1) & 12 & r \\
\hline () & I8 avril $105^{6}$ & & 21 & I 8 & 10 \\
\hline P & Is avril $195^{\circ}$ & & 20 & 3 & 2 \\
\hline 0 & I8 avril 1956 & & 20 & 20 & 14 \\
\hline k & I8 avril $195^{6}$ & & 20 & 20 & 17 \\
\hline
\end{tabular}

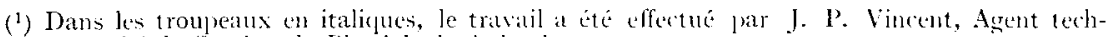
nique principal à la Station de Physiologie Animale.

En outre, nous avons constaté qu'à la baisse d'activité spermatogénétique qu'ont observée OrTavant et 'Thibaudit (I954) s'ajoute une diminution dans l'ardeur du mâle, soit pour chercher les brebis en ostrus, soit même pour s'accoupler (troupeau A notamment). Le comportement sexuel des béliers subirait des fluctuations saisonnières, les béliers ayant une ardeur minima vers le printemps. I a détection des brebis en rut à cette saison de l'année nécessite d'ailleurs comme nous avons pu l'observer dans le troupeau d'expérimentation de la Station, beaucoup plus de temps au printemps qu'à l'automne, l'observation du comportement des brebis 
en rut par un berger expérimenté suppléant quelquefois l'ardeur très faible des béliers bout-en-train.

D'autre part, comme l'indique le graphique $\mathrm{I}$, et comme le montre le graphique 2 (troupeaux I) et E) , le rut apparaît surtout 2 à 3 jours après la fin du traitement et se trouve concentré sur peu de jours.

Il en découle une difficulté importante. En effet, les brebis ayant pour la plupart un rut groupé sur 3-4 jours, il est nécessaire que l'éleveur dis-

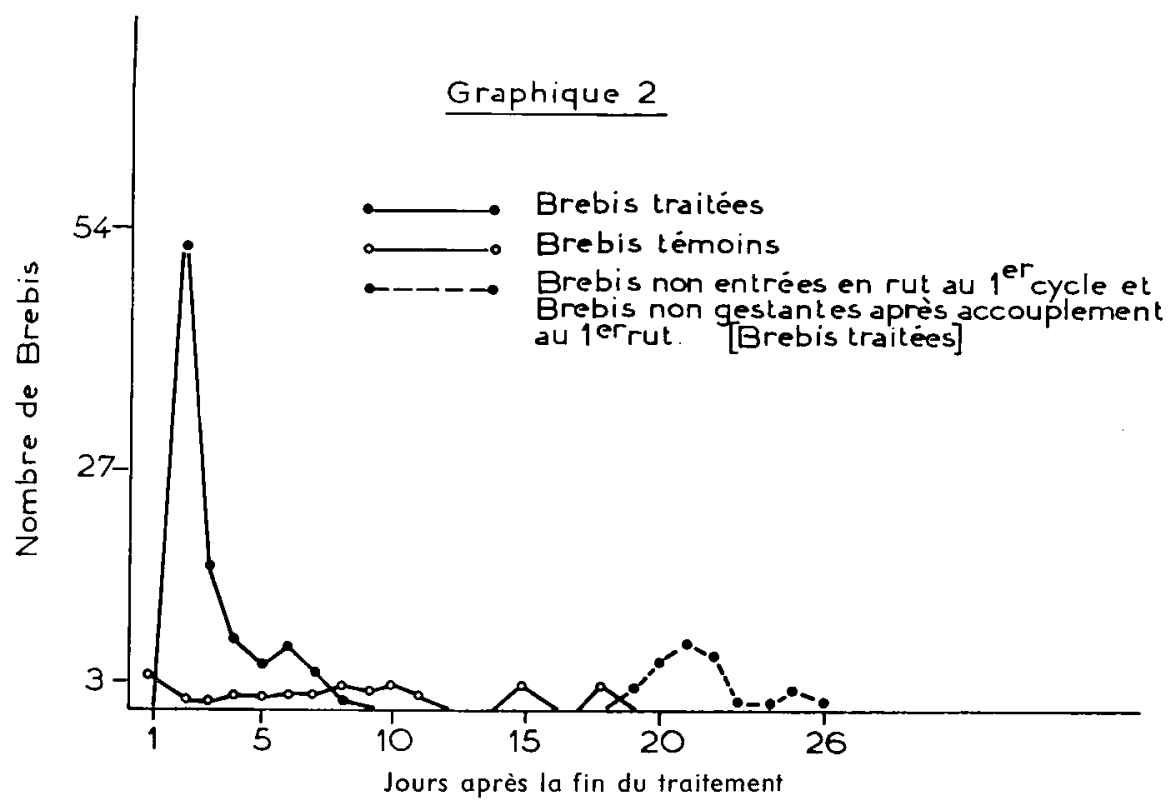

pose d'un nombre de béliers nettement supérieur à celui qui lui serait indispensable pour la lutte de brebis dont les chaleurs s'échelonnent sur un cycle œstrien, soit I6-I 7 jours. Une seule possibilité serait offerte dans ces conditions à l'éleveur qui ne dispose que d'un nombre de béliers normalement en rapport avec l'effectif des brebis, c'est l'insémination artificielle qui à partir d'un même éjaculat permettrait la fécondation de plusieurs brebis.

b) En prenant comme critère d'efficacité le nombre de brebis agnelant on doit remarquer que même lorsque les chaleurs ont été détectées correctement et qu'un pourcentage élevé de Brebis ont pu être accouplées après le traitement, certains résultats restent mauvais : troupeau $\mathrm{F}$ par exemple.

Il semble que l'on puisse attribuer ces échecs à deux causes principales:

IO I'ERTILITÉ DU BÉLIER : I a première cause d'échec serait inhérente

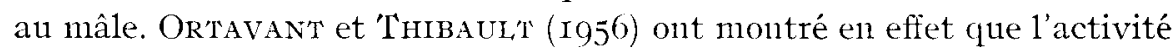
spermatogénétique subit des variations saisonnières parallèles aux variations observées chez les brebis. I'activité testiculaire passe par un minimum en été, ce qui expliquerait partiellement les échecs enregistrés. 
Bien qu'Ortavant et 'Thibault n'aient pu observer de différences entre le pouvoir fécondant de béliers placés en jours longs et en jours courts (communication personnelle), on peut penser qu'une utilisation des béliers beaucoup plus intensive que celle utilisée par ces auteurs affecte leur fertilité.

$2^{\circ}$ Momext DU IRAITEMEN' : I 'ardeur sexuelle ou la fertilité des Béliers à contre-saison ne serait pas le seul facteur en cause. İn effet, il semble que le traitement, quand viennent de cesser les cycles sexuels, au début de l'anostrus, donne des résultats décevants dans le déclenchement du rut (Dauzier et Matríox, non publié). Ces résultats sont cependant trop fragmentaires pour en tirer actuellement des conclusions et des essais systématiques sont en cours, pour déterminer l'intervalle entre la fin de la période sexuelle et l'application du traitement.

\section{RÉSUMÉ}

I, groupage du rut chez la Brebis par voie hormonale, s'il est réalisable chez différentes races à des fins expérimentales, ne peut être utilisé dans des conditions pratiques de l'élevage, par suite d'un nombre d'interventions trop élevé.

Le déclenchement des cycles sexuels féconds pendant une grande partie de la période d'anœstrus, sauf peut-être pendant une courte période réfractaire aussitôt après la fin de la saison sexuelle,permet, d'ores et déjà, un travail de recherches sur la reproduction et la lactation tout au long de l'année.

I'utilisation dans la pratique de l'élevage pose des problèmes supplémentaires non résolus, liés en particulier à l'ardeur sexuelle des Béliers et. à leur fécondité à contre-saison.

\section{RÉFERFNCES BIBLIOGRAPHIQUHS}

Dauzier ( . .), Ortavant (R.), 'Thibault (C.), Wintenberger (S.). - Re. cherches expérimentales sur le rôle de la progestérone dans le cycle sexuel de la Brebis et de la Chèvre. Ann. Endocrinol., 14, 553-559, r953.

Dauzier (L.), Ortavant (R.), 'Thibaut'T (C.) , Wintenberger (S.). - Résultats notveaux sur la gestation à contre-saison chez la Brebis et chez la Chèvre. Ann. Zootech., $3^{\mathrm{e}}$ année, 89-94, I954.

DaUzier (L.), Wintenberger (S.). - Déclenchement simultané de l'œstrus dans un lot de Brebis avec possibilité de gestation ultérieure. Ann. Zootech., $2^{\mathrm{e}}$ année, $49-52$, $195^{2}$.

DUT' (R. H.). -- Induction of oestrus and ovulation in anestrual ewes by use of progesterone and pregnant Mare sertm. J. Amer. Sci., 12, 513$523,1953$.

Dutt (R. H.), Casida (L. E.). - Alteration of the estrual cycle in sheep by use of progesterone and its effect upon subsequent ovulation and fertility. Endoc., 43, 208-217, r943. 
Neilior (J. E.), ColE (H. H.). - The control of estrus in the beef heifer by use of progesterone and gonadotropins. J. Anim. Sci., 15, 650-66r, I 956 .

Gordon (I.) - The use of progesterod and serum gonadotrophin (P. M. S.) in the control of fertility in sheep $J$. A gric. Sci., 50, I23-I5I et I52-I97, I 958 .

O'Mary (C. C.), Pope (A. L.), Casida (L. E.). - The use of progesterone with synchronisation of the estrual periods in a group of ewes and the effect on their subsequent lambing records. J. Anim. Sci., 9, 499-503, I950.

Ortavant (R.), Thibault (C.). - - Influence de la durée d'éclairement sur les productions spermatiques du Bélier. C. R. Soc. Biol., 150, 358 .

Robreson ('T. J.). - Fertility of anoestrous ewes following injection of progesterone and pregnant mare serum (P.M.S.). Austr. J. Agric. Res., 5, 730-736, I954.

RobINson ('T. J.). - Endocrine relationship in the induction of oestrus and ovulation in the anoestrous ewe. $J$. A gric. Sci., 46, 37-43, I955.

'Thibaurt (C.), Dauzier (L.). - Utilisation des hormones sexuelles et des hormones gonadotropes dans l'accroissement de la fécondité chez les Mammifères domestiques. $2^{\circ}$ Cong. Mond. Fert. Stér. Naples, mai 1956 (sous presse). 